Hilson, G. R. F. \& Elek, S. D. (1959). J. gen. Microbiol. 21, 208-220.

\title{
An Investigation into the Development of Gram-negative rods in Penicillin-treated Cultures of Staphylococcus aureus
}

\author{
By G. R. F. HILSON AND S. D. ELEK \\ St George's Hospital Medical School, University of London, Knightsbridge, \\ London, S.W. 1
}

SUMMARY: Attempts were made to obtain Gram-negative rods from the Oxford strain of Staphylococcus aureus in the presence of benzylpenicillin. Two such isolations were made in 476 test cultures and one in 192 controls. The difference is not significant. No reversion to coagulase-positive staphylococci could be demonstrated of a rodshaped Gram-negative organism previously reported as showing this effect. The question of the presence of Gram-negative rods in staphylococcal cultures exposed to penicillin is discussed and it is considered that the phenomenon is due to contamination.

The isolation of Gram-negative rods from a strain of Staphylococcus aureus serially subcultured many times in the presence of increasing concentrations of penicillin was first reported by Klimek, Cavallito \& Bailey (1948). Similar observations were described by Gale \& Rodwell (1949) and by McVeigh \& Hobdy (1952). More recently, it was reported that Gram-negative rods could be found at various times during the development of individual cultures of $S$. aureus in the presence of benzylpenicillin, cephalosporin $\mathrm{C}$ or bacitracin (Crawford \& Abraham, 1957), and this phenomenon was studied further, with special precautions against contamination, by Briggs, Crawford, Abraham \& Gladstone (1957). They used only benzylpenicillin and were able to isolate Gram-negative rods with some regularity from overnight cultures, even when the cultures had been derived from single-cell isolations of the staphylococcal strain. Single-cell isolations were also made of the Gram-negative rods, and during subsequent maintenance of these cultures under different conditions Gram-positive cocci resembling the original strain were once more isolated from them.

There can be no doubt that it is possible to isolate Gram-negative rods from penicillin-treated cultures of Staphylococcus aureus: the finding is, however, open to two interpretations. One is that contamination becomes manifest through the selective action of the drug against the original inoculum; the second, that the rods are in some way directly derived from the staphylococci by mutational or adaptive mechanisms. In view of the special precautions taken by Briggs et al. and their report of the reappearance of staphylococci in bacillary cultures, their work provides the strongest evidence hitherto that the second interpretation may be the correct one. In view of the fundamental importance of the problem an attempt to confirm their findings was made. 


\section{METHODS}

Bacterial strains. Freeze-dried cultures of the Oxford strain of Staphylococcus aureus (NCTC 6571) and of one substrain of a Gram-negative bacillus ('G-11' of Briggs $e t$ al.) were kindly sent by Miss Briggs.

Media. The basic medium used throughout was Bacto (Difco) heart infusion broth (HIB). This was used instead of the tryptic digest broth referred to originally by Briggs $e t$ al. since we learnt that in their later work more frequent isolations of Gram-negative rods occurred in HIB (Briggs, personal communication). For solid media, HIB was used with $2 \%(\mathrm{w} / \mathrm{v})$ New Zealand agar added (HIA). Salt agar was made by adding $7 \%(\mathrm{w} / \mathrm{v})$ sodium chloride to HIA; for a small number of experiments a stock 'saturated salt broth' was made by saturating HIB at room temperature with sodium chloride, allowing the crystals to settle, and using the supernatant. Various conventional media and methods were used in studying the properties of Gram-negative rods: horse blood agar, MacConkey's and Koser's media and peptone water fermentation bottles were prepared according to Mackie \& McCartney (1953) and tests

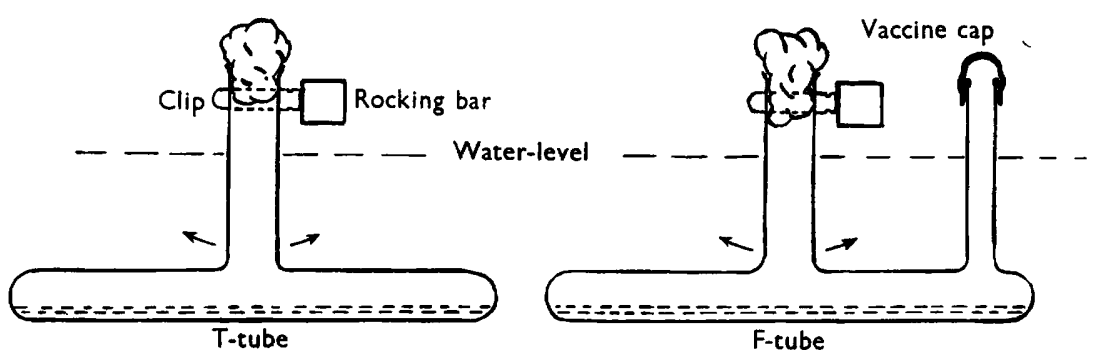

Fig. 1

for indole and hydrogen sulphide production, and Methyl-red and VogesProskauer reactions were carried out as described in the same text. Ammonia production was tested for as described in Topley and Wilson's Principles (1955). Anaerobic cultures were incubated in McIntosh and Fildes jars. Total bacterial counts were made by examining a suitable dilution of a culture in an 'Improved Neubauer' counting chamber; for viable counts the method of Miles \& Misra (1938) was used. For most of the work, HIB was distributed in $9 \mathrm{ml}$. amounts in $25 \mathrm{ml}$. screw-capped bottles ('universal containers'), in 'Pyrex' glass ' $T$ '-tubes (Crawford \& Abraham 1957), or in a modification of the latter referred to as ' $F$ '-tubes (Fig. 1). The latter maintained cultural conditions identical with those in T-tubes but permitted inoculation by syringe and needle through a rubber vaccine cap and so obviated withdrawal of the cotton-wool plug at any time after sterilization. The metal caps of the bottles were also perforated to allow entry with a syringe needle through the rubber disks beneath.

Sterilization of bottles and tubes was by autoclaving at $120^{\circ}$ for $15 \mathrm{~min}$. The media were always sterilized in the containers in which they were to be used. 
The central mouths of the T- and F-tubes were closed by means of a cottonwool plug, and during autoclaving, sterility testing, and storage were covered by brown paper caps held on with rubber bands. All media were tested for sterility by incubation at $37^{\circ}$ for 2 days and then at room temperature for at least 2 days. Syringes were tested for good fit of plungers, lubricated with liquid paraffin, and placed, with needles mounted, in test tubes or boiling tubes, being sealed at the necks by several layers of surgical gauze bandage wrapped around the barrel of the syringe. They were sterilized in the hot-air oven by exposure to $160^{\circ}$ for $1 \frac{1}{2} \mathrm{hr}$.

Inoculation of T-tubes and bottles was by platinum loop or sterile graduated pipettes, except as indicated below. Where a 'closed' technique was being followed, with inoculation by syringe and needle, the following procedure was adhered to: the rubber surface was rubbed liberally with spirit $(95 \%$ ethanol + $5 \%$ water), which was then ignited and the needle passed through the dying flame and into the rubber. To minimize damage to the rubber, no needles thicker than no. $15(0.7 \mathrm{~mm}$. diam.) were used.

Shaking of cultures. The term 'aerated' is used to describe cultures in $\mathrm{T}$ - or F-tubes which were rocked at $\mathbf{4 2}$ cycles/min. through an arc of approximately $70^{\circ}$, partially submerged in a water bath at $37^{\circ}$; 'rotated' cultures were those clipped to a turntable set at $45^{\circ}$ to the vertical, turning at 30 r.p.m. and incubated in a $37^{\circ}$ incubator; and 'stagnant' cultures were those kept in a $37^{\circ}$ incubator without any agitation.

Penicillin solutions. Benzylpenicillin (Glaxo Laboratories Ltd.) was used as the sodium salt. It was dissolved in distilled water and diluted to a concentration of $100 \mu \mathrm{g} . / \mathrm{ml}$. for the earlier attempts to isolate Gram-negative rods. This solution was sterilized by passage through a ' 5 on 3 ' sintered glass filter and distributed by conventional methods in $2 \mathrm{ml}$. amounts in sterile bijou ( $5 \mathrm{ml}$.) screw-cap bottles. The filter was tested by subsequently passing through it $10 \mathrm{ml}$. of an overnight culture of a highly penicillin-resistant strain of Staphylococcus aureus with an excess of fresh broth and incubation for 2 days at $37^{\circ}$; no growth resulted. In the later 'closed' experiments a solution of $20 \mu \mathrm{g} . / \mathrm{ml}$. was filtered through a Seitz EK filter (similarly tested subsequently) and distributed in $8 \mathrm{ml}$. amounts by removal through a rubber diaphragm with a syringe into sterile screw-cap bottles. The solutions were stored at $-15^{\circ}$, a sample bottle being taken occasionally for titration of its penicillin content to detect any deterioration.

Isolation of Gram-negative rods. Penicillin-treated staphylococcal cultures showing abnormal turbidity (all were slightly turbid) were examined by Gram staining and subcultured with a platinum loop on to HIA plates containing $1 \mu \mathrm{g}$. penicillin $/ \mathrm{ml}$. The latter were incubated for $48 \mathrm{hr}$. if necessary. Individual colonies developing on penicillin-agar plates were picked off on to penicillinfree plates and purified by two repetitions of the conventional single-colony subculture. Gram staining and testing for penicillin-resistance by streak inoculation on to penicillin agar completed the preliminary examination, and several HIA slopes were inoculated, incubated and stored at $4^{\circ}$ for further investigations. The other fluid cultures showing only normal turbidity were 
similarly subcultured on to penicillin agar before being discarded. They were not examined routinely by Gram staining since it was very soon found that the short diplobacillary form of the G-11 strain of Briggs et al. (1957) could not be reliably distinguished from the many Gram-negative and swollen diplococci present in penicillin-treated cultures of staphylococci.

Erythromycin and bacitracin sensitivities of $G$-11. These were compared with those of the Oxford staphylococcus by means of a disk diffusion method.

\section{RESULTS}

\section{Isolation of Gram-negative rods from penicillin-treated staphylococcal cultures}

The freeze-dried culture of the Oxford staphylococcus was suspended in $0.25 \mathrm{ml}$. HIB, and a loopful plated on HIA for purification. The remainder of the suspension was spread on a penicillin agar plate. All colonies developing after overnight incubation on HIA resembled Staphylococcus aureus, though poorly pigmented, and all those tested were composed of Gram-positive cocci and gave the slide coagulase reaction. Two successive single colony subcultures were made to complete the purification, and then a colony was subcultured on to several HIA slopes to provide stock cultures for the experiments described below. These cultures were not opened after inoculation until required for use, when they were checked by Gram staining and coagulase testing. The penicillin agar plate inoculated with the remainder of the freezedried culture was sterile after 4 days' incubation at $37^{\circ}$, showing that there had been no admixture with organisms resembling G-11.

The general pattern of inoculation and incubation was the same for all three groups of experiments. In preparation for each day's experiment, a stock culture on HIA was inoculated into a bottle of HIB and incubated overnight. From this stagnant culture sets of 12 or 24 containers of HIB (differing in each group) were inoculated and incubated with agitation at $37^{\circ}$ to an arbitrary standard turbidity, when penicillin was added and the incubation continued overnight. After further stagnant incubation for $48-72 \mathrm{hr}$. they were tested for the presence of Gram-negative rods. The required degree of turbidity before penicillin addition was determined by naked-eye comparison with a 'standard' provided by Miss Briggs. This consisted of one of her cultures, from a similar experiment at the same stage, killed and preserved with a few drops of formalin. It contained about $2 \times 10^{7}$ staphylococci per $\mathrm{ml}$. A few cell counts were done on growing cultures which had reached this degree of turbidity and these also gave results of the same order of magnitude.

Experimental group 1-'rotated cultures'. In this group, the stagnant overnight culture was inoculated into screw-cap bottles of HIB with a graduated pipette in amounts varying from 0.1 to $0.5 \mathrm{ml}$. in different batches of 12 . The periods of incubation with rotation to standard turbidity varied from 1.75 to $2 \cdot 25 \mathrm{hr}$. Penicillin $(100 \mu \mathrm{g}$. $/ \mathrm{ml}$.) was added in $0.1 \mathrm{ml}$. amounts, also with a pipette, to give a final concentration of $1 \mu \mathrm{g} . / \mathrm{ml}$.

After overnight incubation with rotation, lysis by penicillin had produced a moderate reduction in turbidity of the cultures which changed little during 
the subsequent stagnant incubation. Gram staining at this stage showed a mixture of Gram-positive and Gram-negative diplococci with some swollen forms; as already mentioned, these abnormal staphylococci could not be reliably differentiated by their appearance from the short diplobacilli often seen in actively-growing cultures of G-11. Cultures on HIA invariably showed large numbers of surviving staphylococci. In all, none of 144 broth cultures carried out in this manner showed abnormal turbidity, nor did they yield Gram-negative rods on penicillin agar.

This cultural technique differed a little from that of Briggs et al. (1957): its suitability for allowing the emergence of organisms resembling G-11 was subsequently tested. Identical batches of 12 cultures were set up, inoculated with Staphylococcus aureus and treated with penicillin as before, but with the addition, either at the same time as the penicillin or $1 \mathrm{hr}$. later, of a very small inoculum, standardized by total and viable counts, from a young culture of G-11: usually about $0.1 \mathrm{ml}$. of a $10^{-7}$ dilution. In five different batches the estimated average viable inoculum varied from $0 \cdot 3$ to 1.0 bacillary unit; of the total of 60 cultures, 36 grew G- 11 on subsequent incubation, showing that this method of culture would permit the emergence of small numbers of this strain.

Experimental group 2-'aerated cultures'. In view of the failure of the first group of experiments to yield Gram-negative rods an attempt was made to follow the methods of Briggs et al. (1957) in every minute detail affecting cultural conditions. Batches of 12 T-tubes were inoculated with 0.2 or $0.3 \mathrm{ml}$. of overnight cultures, rocked in the water bath to the required turbidity, and treated with penicillin as before.

(a) Four batches of cultures, a total of 48 T-tubes, were tested. Abnormal turbidity was seen in one culture after the overnight aerated incubation, and a Gram-negative rod was isolated on the penicillin-agar plate from this culture. This organism was designated ' $\mathrm{G}-\mathrm{A}$ ' and investigations on it are reported below. Up to this point, since test cultures had yielded no Gram-negative rods, negative controls had not been used; it had been realized, however, that they would become necessary in the face of a positive finding. Four batches of T-tubes of HIB were therefore processed in such a way as to mimic every procedure used in the test cultures, except that the staphylococcal inoculum was lacking: a bottle of HIB was 'inoculated' with a sterile platinum loop (instead of from a staphylococcal stock culture), and incubated overnight; from it, $0.2 \mathrm{ml}$. amounts were added to each T-tube, which, after the usual interval of aerated incubation, received its due amount of penicillin and further incubation. Such control tubes were, of course, normally crystal clear throughout and subcultures were sterile. However, one tube showed a slight turbidity at the end of the full period of incubation and Gram staining and culture on penicillin agar revealed a second Gram-negative rod ' $G-B$ '.

(b) Further sterile precautions appeared to be desirable. At this time, 'settle plates' of HIA exposed to the air in the room in which the T-tubes were being inoculated showed an occasional colony resembling G-B, although this and other Gram-negative bacillary cultures were never handled in the room. 
It was thought likely that contamination might occur through air-borne organisms settling on, or near, the cotton-wool plugs at any time after removal of the protective brown-paper cap, and being sucked into the tube when the plug was removed for addition of the penicillin. A modification of procedure was therefore devised whereby the cotton-wool plug was covered with a sterile metal cap ('oxoid') immediately the brown-paper cover was removed. To introduce a pipette into the tube, the operator lifted off the cap by hand, and an assistant pulled out the plug with sterile forceps, this procedure being reversed after pipetting had been completed. After the penicillin had been added, only the plug was replaced, after flaming. Since the cap fitted loosely, and was in place only during the short period before penicillin addition, there was no significant effect on the amount of aeration of the cultures. Size of inoculum, period of preliminary incubation, and amount of penicillin added to each tube were as in $(a)$. Batches of 12 were set up at a time, and these were alternated with batches of negative controls which had received an 'inoculum' of sterile broth instead of staphylococci. In all, 132 test cultures, and 144 controls, were completed. All of the latter remained sterile; one tube in the ninth batch of the test series yielded a culture of Gram-negative rods on penicillin agar, though it had shown no excess turbidity at $48 \mathrm{hr}$. This organism was labelled G-C.

Experimental group 3-'aerated cultures' with 'closed' technique. In order to diminish further the risks of contamination the preparation of materials for use in this series and the methods of handling the cultures themselves differed considerably from what has been described in regard to the first two series.

Stock cultures of the Oxford staphylococcus: a screw-capped bottle of HIB was inoculated by means of a platinum loop from a stock culture on an HIA slope, with the least possible exposure to the open air. After overnight incubation at $37^{\circ}$, stock broth cultures were made from it by taking out $0.8 \mathrm{ml}$. with a $1 \mathrm{ml}$. syringe and injecting $0.1 \mathrm{ml}$. amounts into broth bottles, which were then incubated overnight and stored at $4^{\circ}$. The remainder of the original broth culture, about $8 \mathrm{ml}$. in volume, was centrifuged at $4000 \mathrm{~g}$ for half an hour and the entire deposit spread on an agar plate containing $1 \mu \mathrm{g}$. penicillin/ml.: no growth occurred after $48 \mathrm{hr}$, at $37^{\circ}$ and 3 further days at room temperature. The overnight broth culture required for each batch of tests was made from a stock broth culture with syringe and needle as described. The inoculation of F-tubes was carried out with a $5 \mathrm{ml}$. syringe and needle, each tube being entered through the rubber cap and receiving $0.2 \mathrm{ml}$. of overnight culture. After the tubes had been inoculated from the latter, it was checked for the presence of Gram-positive cocci and coagulase activity. Benzylpenicillin solution $(20 \mu \mathrm{g} . / \mathrm{ml}$.), was prepared and distributed as described in 'Methods'. For each batch of cultures, $6 \mathrm{ml}$. of penicillin solution was taken from a bottle with a $10 \mathrm{ml}$. syringe, and $0.5 \mathrm{ml}$. injected into each F-tube, the final concentration being once more $1 \mu \mathrm{g} . / \mathrm{ml}$.

These precautions were intended to ensure that the materials used in the tests, once sterilized, or, in the case of the staphylococcal cultures, tested for 
the absence of Gram-negative rods, should not suffer exposure to the open air greater than that occasioned by the presence of a capillary meniscus of fluid inside a syringe needle. The general pattern of testing was as before, batches of 12 F-tubes being inoculated, incubated in the water bath with aeration for about $2 \mathrm{hr}$., and injected with penicillin before further aerated and stagnant incubation. No control tubes were set up in this series. None of 152 cultures yielded Gram-negative rods.

\section{Characteristics of strains $G-11, G-A, G-B$ and $G-C$}

Strain $G$-11. The freeze-dried culture of this provided by Miss Briggs was re-constituted with a few drops of HIB and plated out on HIA. After overnight incubation at $37^{\circ}$ it was possible to detect the fact that two types of colony were present. Both types were about $2 \mathrm{~mm}$. diameter, smooth and creamy in appearance, and butyrous in consistency, but one was very slightly more translucent than the other. After standing at room temperature for a further $24 \mathrm{hr}$., distinction between the colonial types was easier, as the more translucent ones showed a moderate peripheral mucoid change ('Schleimwallbildung'); the other type of colony had merely become slightly more opaque. The translucent colonies predominated and were made up of Gram-negative diplobacilli; the other colonies were those of a staphylococcus giving a positive slide coagulase reaction and with a penicillin-sensitivity similar to that of the Oxford staphylococcus. Three serial conventional single-colony subcultures on HIA of the Gram-negative organism produced growths having all the appearances of a pure culture, in which colonies containing Gram-positive cocci could no longer be found. A single colony was used to inoculate several HIA slopes for stock cultures of G-11. Most of the properties investigated are listed in Table 1. The main points of note are that it is a freely growing non-motile Gram-negative rod which is an obligate aerobe, grows on MacConkey's medium, is non-saccharolytic and utilizes citrate in Koser's medium. Its properties in relation to penicillin are not shown in the table but are of some interest.

Penicillin sensitivity. Serial doubling dilutions of penicillin in HIB were made (4 ml. volumes in universal containers) and each inoculated with one drop of an overnight HIB culture of G-11. After 24 hr. incubation at $37^{\circ}$ growth equal to the control took place in $4 \mu \mathrm{g} . / \mathrm{ml}$. with partial growth in $8 \mu \mathrm{g} . / \mathrm{ml}$. At $48 \mathrm{hr}$. full growth had extended to $16 \mu \mathrm{g}$. $/ \mathrm{ml}$. To investigate the effect of inoculum size, serial tenfold dilutions of a young (incubated for $4 \mathrm{hr}$. and faintly turbid) HIB culture of G-11 were made, and HIA plates containing $16,8,4,2$ and $0 \mu \mathrm{g}$. penicillin $/ \mathrm{ml}$. were inoculated with $0.02 \mathrm{ml}$. drops of each dilution. Each plate also received a loopful of undiluted culture and a spot inoculum from a slope culture of the Oxford staphylococcus as a control of penicillin activity. After 3 days' incubation, growth from the various dilutions was equal to the control on concentrations of penicillin up to $4 \mu \mathrm{g} . / \mathrm{ml}$., but there was none on plates containing higher concentrations. The loopful of undiluted culture, however, produced growth on the $16 \mu \mathrm{g} . / \mathrm{ml}$. plate.

Penicillinase production. This 'inoculum effect' suggested penicillinase 
production, and a penicillin-destroying activity of G-11 was in fact demonstrated. A heavy inoculum of the strain was streaked in the centre of a HIA plate containing $1 \mu \mathrm{g}$. penicillin $/ \mathrm{ml}$. and incubated overnight, producing a streak of luxuriant growth. A loop carrying a portion of a solid culture of the Oxford staphylococcus was then streaked at right angles up to and through the G-11 growth with a steady movement. After further overnight incubation, growth of the staphylococcal inoculum was found to a zone extending $3 \mathbf{m m}$. out from the G-11 streak, demonstrating considerable reduction in effective

Table 1. Properties of the Oxford staphylococcus and of the

Gram-negative organisms $G-11, G-A, G-B$ and $G-C$

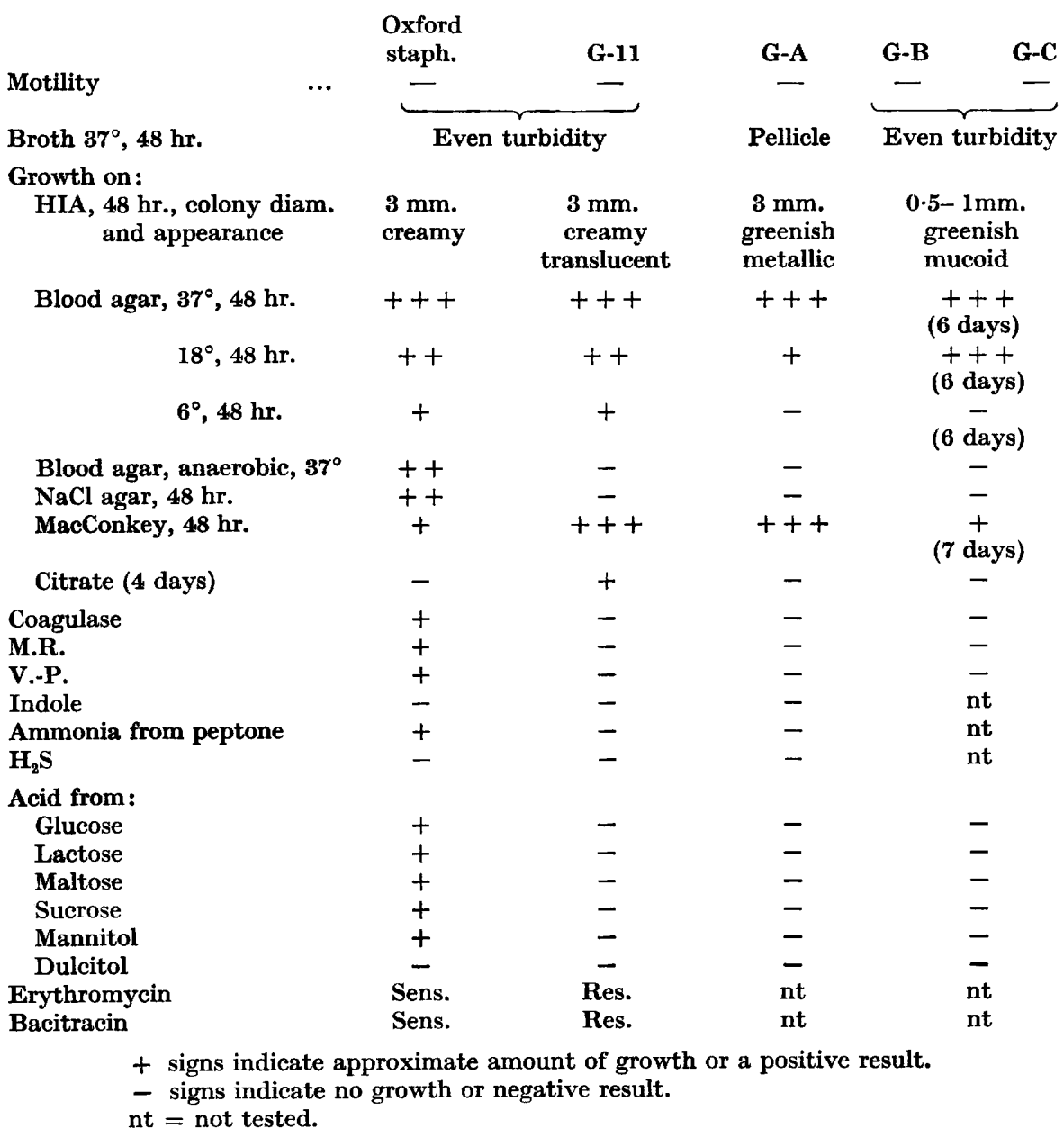

penicillin concentration near to the latter. To confirm this finding, $10 \mathrm{ml}$. HIB containing $8 \mu \mathrm{g}$. penicillin $/ \mathrm{ml}$. was inoculated with $0.1 \mathrm{ml}$. of a fully grown culture of G-11 in HIB and incubated overnight. The densely turbid culture was centrifuged to deposit most of the bacilli, and the supernatant inoculated 
with the Oxford staphylococcus. Free growth of the latter on further incubation, as shown by Gram staining and subculture on salt agar, demonstrated penicillin destruction by G- 11 .

Strain $\boldsymbol{G}-\boldsymbol{A}$. This organism was isolated from an aerated staphylococcal culture of experimental group 2a and was a non-motile Gram-negative rod with a colonial appearance on HIA very similar to that of G-11. Its characteristics are also described in Table 1; it differed from G-11 in producing slightly greenish metallic colonies on HIA, a stringy growth and pellicle in HIB, instead of a diffuse turbidity, and in not utilizing citrate.

Strains $\boldsymbol{G}-\boldsymbol{B}$ and $\boldsymbol{G}-\boldsymbol{C}$. G-B was isolated from an aerated broth control tube of experimental group 2a, and G-C from a penicillin-treated staphylococcal culture of group $2 \mathrm{~b}$. These organisms appeared very similar to each other, and to have several characters (Table 1) contrasting markedly with those of G-11 and G-A. Both were slowly growing non-motile Gram-negative rods, producing greenish mucoid colonies reaching $2 \mathrm{~mm}$. diameter on blood agar only after 6 days and growing just as well at room temperature $\left(18^{\circ}\right)$ as at $37^{\circ}$.

\section{Attempts to recover staphylococci from cultures of $G-11$}

It has already been mentioned that the reconstituted freeze-dried culture of G-11 gave rise to a mixture of Gram-negative rods and a penicillin-sensitive coagulase-positive staphylococcus. However, in the course of routine purification, the first subculture of a colony of the Gram-negative organism showed only two colonies of Gram-positive cocci and each of two further subcultures showed none. From the last of these, stock HIA slopes of G-11 were set up, and in the course of investigations involving the use of this organism, many further subcultures on HIA and in HIB were made. Gram-positive cocci were not found in any of these.

The selective action of sodium chloride was also used in order to try to discover the presence of staphylococci in cultures of G-11. Preliminary experiments showed that G-11 was inhibited, with some sterilization, by sodium chloride in approximately $8 \%(\mathrm{w} / \mathrm{v})$ concentration in HIB, while small inocula of the Oxford staphylococcus grew readily under these conditions. Batches of 12 screw-capped bottles of HIB $(10 \mathrm{ml}$.) were inoculated with $0.2 \mathrm{ml}$. of an overnight broth culture of G-11 and rotated for $2 \frac{1}{2} \mathrm{hr}$. at $37^{\circ}$. To each of the moderately turbid cultures $3 \mathrm{ml}$. of sterile saturated salt broth was then added with a graduated pipette, and incubation with rotation continued overnight. A loopful was taken from each culture on to salt agar plates which were incubated for $48 \mathrm{hr}$. Four batches of twelve were completed in this way. In one culture of the first batch dense turbidity had developed after overnight incubation, and this was found to contain a mixture of albus and citreus types of staphylococci, both coagulase-negative, and the latter with optimal growth at room temperature. All of the other 47 cultures were negative. Settle plates of the room in which this work was being carried out showed that similar staphylococci were the predominant aerial flora. 


\section{DISCUSSION}

The results reported above may be summarized as follows: out of 144 agitated, and 332 aerated (total 476), cultures of the Oxford staphylococcus treated with benzylpenicillin in the logarithmic phase of growth, only two yielded Gramnegative rods (G-A and G-C). These differed in a number of properties from each other and from the organism G-11 of Briggs et al. (1957). Of 192 control tubes lacking a staphylococcal inoculum, one yielded a Gram-negative rod (G-B). In approximately a hundred subcultures (after routine purification) of G-11, in a high proportion of which the selective action of sodium chloride was employed, no staphylococci resembling the Oxford strain were found. These findings, therefore represent only a partial confirmation of the work of Briggs et al. (1957) in regard to the apparent conversion of staphylococci to Gramnegative rods, and none in regard to the reversion of the latter to coagulasepositive staphylococci.

It appears to us that the problem of the transformation of a staphylococcus into a Gram-negative rod requires consideration under two headings. The first is whether standard bacteriological techniques, even with the added precautions that have been used, are sufficiently reliable to exclude contamination with certainty. The second involves an assessment of the evidence for a genetic relationship between the two strains.

Bacteriological procedures for the maintenance of pure cultures involve a chain of techniques: a chain whose strength is only that of its weakest link. Heat treatment of media and glassware provides the strongest link, with the least probability of contamination, and that provided by filtration with subsequent testing of the filter must be nearly as strong. It is very difficult to judge the risks of contamination incurred in the transference of the bacterial strain itself, which necessarily is exposed to a variable extent during the process. There is no indication in the reports of earlier workers (Klimek et al. 1948; Bellamy \& Klimek, 1948; Gale \& Rodwell, 1949; Kawamata \& Shigeto, $1955)$ that they used any precautions against contamination beyond those customarily employed. Moreover, since they used methods of serial transfer, it is likely that the risk of contamination in their work was high. They used no controls which would allow any estimate of its probability. With the aid of an inoculation cabinet and other precautions Briggs et al. (personal communication) were able to avoid contamination in approximately 300 controls; in our work one contaminant appeared in 192 control cultures.

We are not convinced that single cell selection by micro-manipulation (de Fonbrune, 1949) lessens the risk of contamination very much. It provides a reasonable guarantee that a culture with properties generally resembling those of the parent has arisen from a single member of the latter, but our own experience with the method suggests that since the growth developing from the single cell is subsequently handled by standard methods, contamination is possible at that and later stages. The 'syringe and rubber diaphragm' method of inoculation used by us in Experimental group 3 seemed on theoretical grounds likely to diminish the risk of contamination, and no rods were found 
in 152 cultures; a very much larger number of tests would however be necessary to establish statistically the advantage of this method.

The success in general of standard methods for the maintenance of pure cultures depends not so much on the absence of contamination, as on the fact that a contaminant can rarely become manifest in the absence of an agent acting selectively in its favour. Conversely, it is common knowledge from work in the field of bacterial genetics that when such selection is applied, a single resistant mutant (or contaminant) can be detected with ease. It is our view that the methods so far employed in this field (including our own) have not been shown to be sufficiently refined to allow contamination to be excluded as an explanation for the emergence of Gram-negative rods from penicillintreated cultures of Staphylococcus aureus. The evidential value of this emergence therefore depends on whether it occurs significantly more frequently in test cultures than in the controls. In our series there was no statistically significant difference. In the earlier work of Briggs et al. (1957), bacilli were found only in the test cultures with none in the controls, but they have been unable to confirm this (Briggs, Crawford, Abraham \& Gladstone, 1959), and Paine \& Daniel (1959) have also been unable to isolate Gram-negative rods.

It is a theoretical possibility that the Gram-negative rods are derived from the staphylococci, but the probability of this has to be viewed in the light of existing knowledge on bacterial genetics. There are no hard and fast rules about the number of characteristics by which a mutant can differ from its parent and still be accepted as such rather than as a new species. However, the resistant mutant isolated after the action of a selective agent is likely to differ in only a few characters, and with each new unrelated character there is a greatly reduced probability of it being a mutant. If the characters are not linked, the probability is the product of the individual mutation rates. When several characters are altered simultaneously (even if their individual rates are high) the mathematical probability of such an occurrence requires a parent population of astronomical size. The greater, therefore, the divergence from the parent, the greater the likelihood that a different species is being investigated: that is, contamination has to be postulated. Briggs $e$ al. (1957) tabulate a series of comparisons between the Oxford staphylococcus and their organism including characteristics of morphology, sugar fermentation, protease and coagulase activity, haemolysin production, phage typability and carriage, and penicillin and polymyxin sensitivities. They differ in almost every particular. Our investigations confirm and supplement these data, findings of note being that G-11 utilizes citrate and shows much greater resistance to erythromycin and bacitracin than the staphylococcus. We also showed G-11 to possess penicillin-destroying activity, a result contrary to the findings of Briggs $e t$ al. and possibly due to differences in the techniques used to demonstrate the effect. Our additions to the list of properties investigated show a further divergence in character between the Oxford staphylococcus and G-11; in fact, it is notable that the staphylococcus and the Gram-negative rod share hardly any positive characters. Numerous explanations have been adduced by previous workers in this field to account for the profound changes apparently 
produced in staphylococci by the action of penicillin: the various considerations outlined above, however, taken together with the failure of Paine \& Daniel (1959), Briggs et al. (1959) and ourselves to repeat completely the work of Briggs et al. (1957) lead us to believe that the simplest and most likely explanation is that of contamination. If this is the case, many of the observations that have been made on the metabolism of such penicillin-resistant organisms are fallacious.

It must be admitted, however, that we are unable to explain the findings of Briggs et al. (1957). In spite of frequent consultations with Miss Briggs and Dr Gladstone we could not point to any flaw in technique sufficient to account for the frequency with which rods appeared in their staphylococcal cultures while the controls remained negative, nor for the 'reversion' phenomena. Moreover, it is not entirely clear why the penicillin-resistant strains reported by different workers have a general resemblance to each other and to the genera Achromobacter or Alcaligenes; the latter are common enough in the environment of the laboratory and the cold-room, but contamination by other equally common species might have been expected. In this connexion the resistant Gram-positive coccus of Kawamata \& Shigeto (1955) may have been a species of micrococcus unrelated to the 'parent staphylococcus' since their criteria for identification were inadequate, and both positive and negative differences in fermentative capacities were shown. Furthermore, certain differences exist between the various Gram-negative organisms described. Our strain fairly closely resembled G-11, but did not utilize citrate; the slowly growing G-C appeared very different indeed. The organism of Klimek et al. (1948) fermented glucose.

In conclusion, while it cannot be dogmatically stated that this form of transformation does not exist, we feel that the onus of proof still remains to show that staphylococci can ever give rise to Gram-negative rods under the conditions reported. It is our belief that contamination is the entire explanation of these phenomena.

It is with great pleasure that we accord our appreciation for the generous help given by Dr G. P. Gladstone and Miss S. Briggs, in providing cultures and enabling us to replicate the minutiae of their technique.

\section{REFERENCES}

Bellamy, W. D. \& Kuimek, J. W. (1948). Some properties of penicillin-resistant staphylococci. J. Bact. 55, 153.

Briggs, S., Crawford, K., Abraham, E. P. \& Gladstone, G. P. (1957). Some properties of Gram-negative bacilli obtained from a strain of Staphylococcus aureus in the presence of benzylpenicillin. J. gen. Microbiol. 16, 614.

Briggs, S., Crawford, K., Abraham, E. P. \& Gladstone, G. P. (1959). Further observations on the relationships between Gram-negative rods and staphylococci grown in the presence of penicillin. J. gen. Microbiol. 21, 205.

Crawford, K. \& Abraham, E. P. (1957). The synergistic action of cephalosporin C and benzylpenicillin against a penicillinase-producing strain of Staphylococcus aureus. J. gen. Microbiol. 16, 604.

de Fonbrune, P. (1949). Technique de micromanipulation. Paris: Masson et Cie. 
Gale, E. F. \& Ropwell, A. W. (1949). The nature of resistance to penicillin in Staphylococcus aureus. J. gen. Microbiol. 3, 127.

Kawamata, J. \& Shigero, T. (1955). Altered growth requirements accompanying penicillin resistance in Micrococcus pyogenes var. aureus. Med. J. Osaka Univ. 6, 107.

Klimek, J. W., Cavallito, C. J. \& Bailey, J. H. (1948). Induced resistance of Staphylococcus aureus to various antibiotics. J. Bact. 55, 139.

Mackie, T. J. \& McCartney, J. E. (1953). Handbook of Practical Bacteriology, 9th ed. Edinburgh and London: E. and S. Livingstone, Ltd.

McVeIGH, I. \& HobDy, C. J. (1952). Development of resistance by Micrococcus pyogenes var. aureus to antibiotics: morphological and physiological changes. Amer. J. Bot. 39, 352.

Mrles, A. A. \& Misra, S. S. (1938). The estimation of the bactericidal power of the blood. J. Hyg., Camb. 38, 732.

Paine, Jun., T. F. \& Daniel, R. R. (1959). Attempts to obtain Gram-negative rods from staphylococci treated with penicillin. J. gen. Microbiol. 21, 203.

Topley and Wilson's Principles of Bacteriology and Immunity (1955), 4th ed. Revised by G. S. Wilson \& A. A. Miles. London: Edward Arnold (Publishers) Ltd.

(Received 9 April 1959) 